\title{
On the structure of strange non-chaotic attractors in pinched skew products
}

\author{
TOBIAS H. JÄGER \\ Mathematisches Institut, Friedrich-Alexander-Universität Erlangen-Nürnberg, Germany \\ (e-mail: jaeger@mi.uni-erlangen.de)
}

(Received 23 February 2004 and accepted in revised form 16 August 2005)

\begin{abstract}
The existence of non-continuous invariant graphs (or strange non-chaotic attractors) in quasiperiodically forced systems has generated great interest, but there are still very few rigorous results about the properties of these objects. In particular, it is not known whether the topological closure of such graphs is typically a filled-in set, i.e. consists of a single interval on every fibre, or not. We give a positive answer to this question for the class of so-called pinched skew products, where non-continuous invariant graphs occur generically, provided that the rotation number on the base is diophantine and the system satisfies some additional conditions. For typical parameter families these conditions translate to a lower bound on the parameter. On the other hand, we also construct examples where the non-continuous invariant graphs contain isolated points, such that their topological closure cannot be filled-in.
\end{abstract}

\section{Introduction}

Generally speaking, for a quasiperiodically forced monotone interval map, invariant graphs play the same role as fixed points, for unperturbed maps. However, in contrast to fixed points, there are two different types of invariant graphs. In the simpler case, such a graph is continuous. If, in addition, it has a negative Lyapunov exponent, a lot of conclusions about its regularity, structural stability, etc, can be drawn (see [Sta99, SS00]). More difficult, but also more interesting, is the case of non-continuous invariant graphs. When their Lyapunov exponent is negative, such graphs are often referred to as strange non-chaotic attractors (SNAs). Their existence has recently received a great deal of attention in theoretical physics, and consequently there are a lot of numerical studies on this topic ([PNR01] gives a good overview and further references). On the other hand, rigorous results are still scarce. The existence of SNAs has only been proved for rather specific systems (see [Her83] for quasiperiodically driven Moebius transformations, [Kel96, BO96] for pinched skew products), and even in these cases there are a lot of open questions regarding their further properties. It is one of these questions that we want to address here. 
Quasiperiodically forced monotone interval maps. In order to give a precise formulation of the problem we want to study, we need a few basic definitions. First of all, a quasiperiodically forced monotone interval map is a continuous map of the form

$$
T: \mathbb{T}^{1} \times I \rightarrow \mathbb{T}^{1} \times I, \quad(\theta, x) \mapsto\left(\theta+\omega, T_{\theta}(x)\right)
$$

where $\omega \in[0,1] \backslash \mathbb{Q}$ is a fixed irrational rotation number, $I \subset \mathbb{R}$ is a compact interval and all of the fibre maps $T_{\theta}$ are monotonically increasing on $I$. An invariant graph for such a system $T$ is a function $\varphi: \mathbb{T}^{1} \rightarrow I$ which satisfies

$$
T_{\theta}(\varphi(\theta))=\varphi(\theta+\omega) \quad \text { for all } \theta \in \mathbb{T}^{1} .
$$

This equation implies that the corresponding point set $\Phi:=\left\{(\theta, \varphi(\theta)) \mid \theta \in \mathbb{T}^{1}\right\}$ is forward invariant, i.e. $T(\Phi)=\Phi$ (not necessarily $T^{-1}(\Phi)=\Phi$, as we did not assume strict monotonicity). On the other hand, whenever $K$ is a compact and forward invariant set, we can define an invariant graph by

$$
\varphi_{K}^{+}(\theta):=\max \{x \in I \mid(\theta, x) \in K\}
$$

(the invariance being a direct consequence of the monotonicity of the fibre maps). Further, as $K$ is compact, $\varphi_{K}^{+}$will be upper semi-continuous. In the same way, a lower semi-continuous invariant graph $\varphi_{K}^{-}$can be defined via the minimum. We call the set $K$ 'filled-in' if $K=\left[\varphi_{K}^{-}, \varphi_{K}^{+}\right]:=\left\{(\theta, x) \mid \varphi_{K}^{-}(\theta) \leq x \leq \varphi_{K}^{+}(\theta)\right\}$.

Particularly interesting for our purposes will be the case where $K$ is the global attractor of the system, defined as $K:=\bigcap_{n=0}^{\infty} T^{n}\left(\mathbb{T}^{1} \times I\right)$. The corresponding bounding graphs will then be called the upper and lower bounding graphs of the system $T$ and be denoted by $\varphi^{+}$and $\varphi^{-}$. (All of this is thoroughly discussed in [Gle02], [Sta03] or [Jäg03].)

The question. Suppose that $\varphi$ is an invariant graph that is not continuous. Its topological closure $\bar{\Phi}$ is a compact and invariant set, again bounded from above and below by invariant graphs $\varphi^{+}$and $\varphi^{-}$. The set $\left[\varphi^{-}, \varphi^{+}\right]$is also a compact invariant set and surely $\bar{\Phi} \subseteq\left[\varphi^{-}, \varphi^{+}\right]$. However, is $\bar{\Phi}=\left[\varphi^{-}, \varphi^{+}\right]$?

This question was already asked by Herman in [Her83, §4.14] for certain quasiperiodically forced Moebius transformations, and then repeated a number of times in different situations (see [Kel96, Gle02, Sta03]).

Results. Here, we address the problem in the setting of so-called pinched skew products first introduced in [GOPY84]. Their particular structure allows us to prove the existence of non-continuous invariant graphs by a few simple and elegant arguments (see [Kel96, BO96]; a slight generalization can be found in [Gle02]). Often, such systems are given by (1.1) with $I=[0, L], L>0$ and fibre maps

$$
T_{\theta}(x)=\alpha g(\theta) f(x),
$$

where $f: \mathbb{R}^{+} \rightarrow \mathbb{R}^{+}$is monotonically increasing with $f(0)=0, g: \mathbb{T}^{1} \rightarrow \mathbb{R}^{+}$has exactly one zero and $\alpha$ is a positive parameter. A typical example would be $f(x)=\tanh (x)$ and $g(\theta)=|\sin (\pi \theta)|$. Note that $f(0)=0$ implies that the lower bounding graph is always $\varphi^{-} \equiv 0$. In short terms, our main result can now be stated as follows (see Corollary 4.3). 
Suppose that $f$ and $g$ satisfy some mild conditions concerning their geometry and regularity (specified before Corollary 4.3) and the rotation number $\omega$ is of diophantine type. Then for all sufficiently large parameters $\alpha$ the upper bounding graph $\varphi^{+}$of the system $T$ defined by (1.4) has the property $\overline{\Phi^{+}}=\left[0, \varphi^{+}\right]$.

As already mentioned, we also give examples where the topological closure of the upper bounding graph cannot be filled-in because the graph contains isolated points. These examples can be chosen smooth with diophantine rotation on the base, showing that the additional assumptions on the system we use to derive the above result are indeed crucial, and cannot be neglected. (See also Remarks 4.6, 5.1 and 5.2 for a further discussion of these examples.)

Overview. In $\S 2$ we briefly sketch the arguments used to establish the existence of noncontinuous invariant graphs in pinched skew products. In order to gain more insight about their creation and structure, in $\S 3$ we introduce the iterated upper boundary lines. This sequence of continuous graphs converges monotonically decreasing to the upper bounding graph, and their shape can be controlled to some extent by putting additional restrictions on the geometry of the system. In $\S 4$ this is then used to derive the mentioned result, and $\S 5$ contains the construction of the counterexamples.

\section{Pinched skew products}

Suppose that a map $T$ as in (1.1) with $I=[0, L]$ for some $L>0$ additionally satisfies

$$
\begin{aligned}
T_{\theta}(0)=0 & \text { for all } \theta \in \mathbb{T}^{1} \quad \text { (invariant 0-line) } \\
T_{\theta} \equiv 0 & \text { for some } \theta \in \mathbb{T}^{1} \quad \text { (pinching). }
\end{aligned}
$$

As mentioned above, (2.1) ensures that the lower bounding graph of such a system is always $\varphi^{-} \equiv 0$. Further, (2.2) implies that any other invariant graph must be equal to zero on a dense subset of $\mathbb{T}^{1}$, namely the forward orbit of $\theta$. Thus, apart from $\varphi^{-}$no other invariant graph can be continuous.

Now suppose that all of the fibre maps $T_{\theta}$ are differentiable and denote the derivative by $D T_{\theta}$. Then by

$$
\lambda(\varphi):=\int_{\mathbb{T}^{1}} \log D T_{\theta}(\varphi(\theta)) d \theta
$$

the Lyapunov exponent of an invariant graph $\varphi$ can be defined. It is easy to show that whenever $T$ satisfies some mild conditions (namely when $\theta \mapsto \inf _{x \in I} \log D T_{\theta}(x)$ is integrable), the Lyapunov exponent of the upper bounding graph cannot be strictly positive (e.g. [Jäg03, Lemma 3.5]). For systems of the form (1.4), a simple computation yields

$$
\lambda(0)=\log f^{\prime}(0)+\log \alpha+\int_{\mathbb{T}^{1}} \log g(\theta) d \theta
$$

whenever $f^{\prime}(0)>0$ and $\log g$ is integrable. This is surely positive for sufficiently large $\alpha$. Thus, the upper bounding graph $\varphi^{+}$cannot be equal to $\varphi^{-}$and must therefore be non-continuous in this case. 

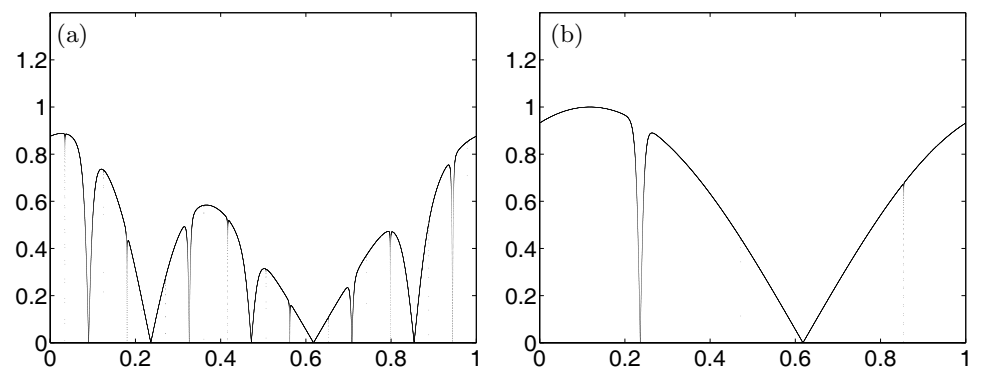

FIGURE 1. Semi-continuous upper bounding graphs in the parameter family $(\theta, x) \mapsto(\theta+\omega, \tanh (\alpha x)$. $|\sin (\pi \theta)|)$. Here $\omega$ is the golden mean. The parameter values are (a) $\alpha=3$ and (b) $\alpha=32$.

Even if they are rather degenerate in some sense, the fact that the existence of strange non-chaotic attractors can be established so easily makes pinched skew products an ideal setting for studying their further properties. The results obtained here may then at least give hints about more general systems. This is further supported by the fact that although invertible systems lack the pinched structure, their minimal sets will still be pinched sets (i.e. consist of only one point on a dense set of fibres, see [Sta03]), and therefore have some similarities with the global attractors of pinched skew products.

\section{The shape of the iterated upper boundary lines}

The argument sketched in the last section gives the non-continuity of the upper bounding graph and ensures that its topological closure contains the 0-line, but apart from that it offers little further information. To explain the shape of such SNAs as depicted in Figure 1, recall that the upper bounding graph was defined via the global attractor $K=\bigcap_{n \in \mathbb{N}} T^{n}\left(\mathbb{T}^{1} \times I\right)$. The sets $K_{n}:=\bigcap_{i=1}^{n} T^{i}\left(\mathbb{T}^{1} \times I\right)$ are bounded above by the iterates of the upper boundary line $\mathbb{T}^{1} \times\{L\}$. To be more precise, define a monotonically decreasing sequence of continuous graphs by $\varphi_{0}: \equiv L$ and $\varphi_{n+1}(\theta):=T_{\theta-\omega}\left(\varphi_{n}(\theta-\omega)\right)$ (alternatively, $\left.\varphi_{n}(\theta):=T_{\theta-n \omega}^{n}(L)\right)$. Then $K_{n}=\left[0, \varphi_{n}\right]$, and the graphs $\varphi_{n}$ converge pointwise and monotonically decreasing to $\varphi^{+}$. It therefore seems reasonable to hope that by understanding the behaviour of the $\varphi_{n}$ we can gain more insight about their limit object.

Figure 2 shows the first six iterates of the upper boundary line. The pictures suggest a strikingly simple scenario. Suppose that $T$ is pinched exactly at $\theta=0$ (i.e. $T_{0} \equiv 0$ and all other fibre maps are strictly monotonically increasing) and let $\tau_{n}:=n \omega \bmod 1$. Then in the $n$th iteration step a new 'peak' appears at $\tau_{n}$. The graphs $\varphi_{n-1}$ and $\varphi_{n}$ differ on a small interval centred around $\tau_{n}$, but apart from that they seem to coincide. In addition, the peaks seem to get steeper at an exponential rate, and accordingly the width of the intervals decays exponentially. Figure 3 briefly explains, on an heuristic level, how such an observation can be used to show that $\Phi^{+}$is indeed dense in $\left[0, \varphi^{+}\right]$. A slightly refined version of this will then determine our strategy in the proof of Theorem 4.1 (see the beginning of $\S 4$ ).

Unfortunately, there are some complications in the general case. When the fibre maps are strictly monotonically increasing, the graphs $\varphi_{n}$ will be strictly monotonically decreasing and thus two of them can never exactly coincide (except on the orbit of the pinched fibre). Still, it is possible to control the behaviour of these graphs, but instead of concentrating on the peaks as our observations suggest we must rather take care of the 

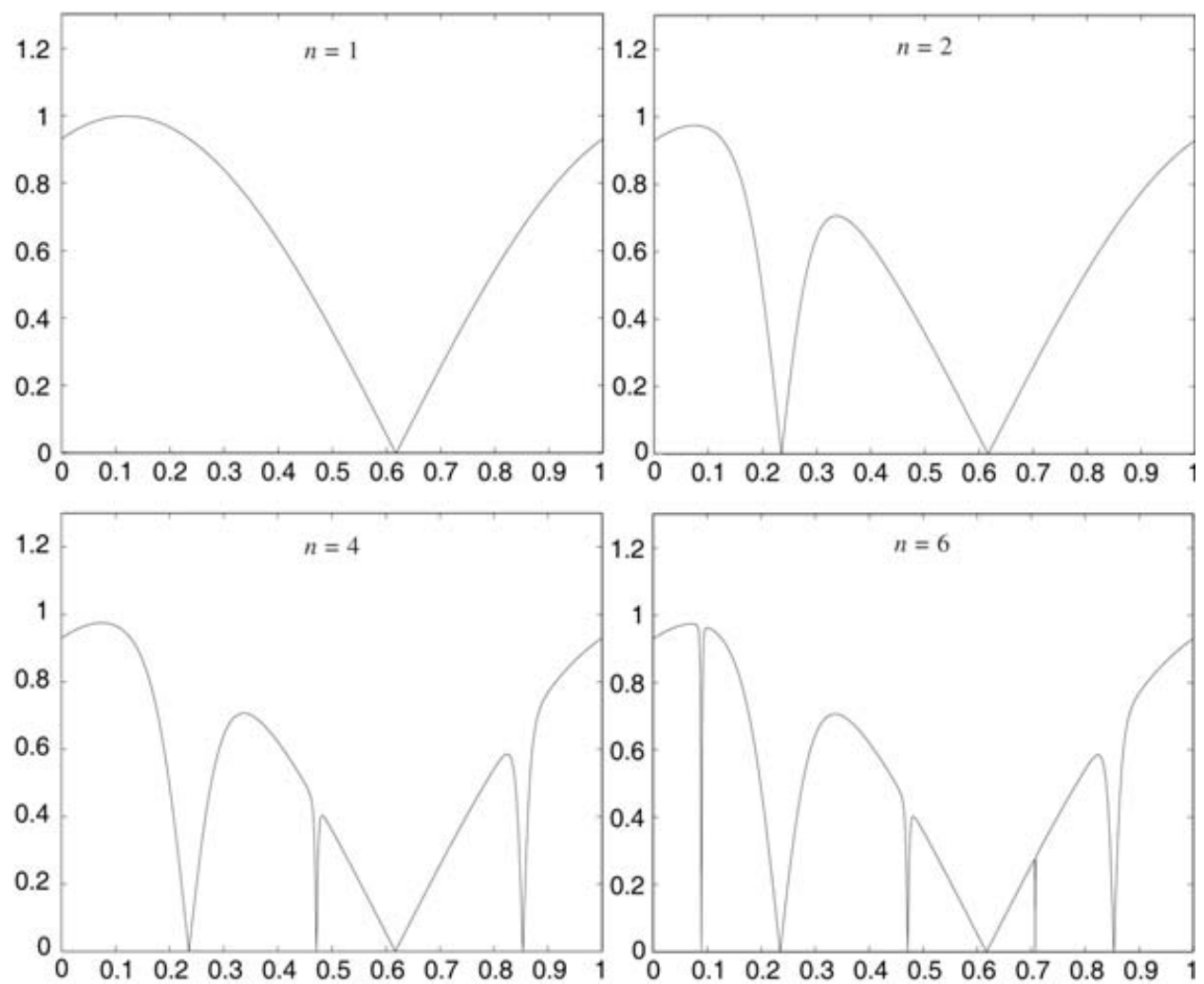

FIgURE 2. The iterated upper boundary lines $\varphi_{n}$ of the system $(\theta, x) \mapsto(\theta+\omega, \tanh (5 x) \cdot|\sin (\pi \theta)|)$. Again $\omega$ is the golden mean.

regions away from the peaks (see Lemma 3.3). However, this will turn out to be sufficient for our purposes.

In order to obtain the required control we must put several restrictions on the systems we study. As mentioned above, for parameter families as in (1.4) these will basically translate to a lower bound on the parameter.

Let $\tau_{n}:=n \omega \bmod 1 \in \mathbb{T}^{1}$ as above and suppose that $\omega \in[0,1] \backslash \mathbb{Q}$ satisfies the diophantine condition

$$
d\left(\tau_{n}, 0\right) \geq c \cdot n^{-d}
$$

for some $c, d>0$. Further, assume that $T$ is a pinched skew product and, for some $\alpha>2$, $\beta>0$ and $\gamma>0$, the following holds $\dagger$ :

$$
\begin{aligned}
D T_{\theta}(x) & \leq \alpha \quad \text { for all }(\theta, x) \in \mathbb{T}^{1} \times I, \\
D T_{\theta}(x) & \leq \alpha^{-\gamma} \quad \text { for all } \theta \in \mathbb{T}^{1}, x \geq 1, \\
\left|\frac{\partial}{\partial \theta} T_{\theta}(x)\right| & \leq \beta \quad \text { for all }(\theta, x) \in \mathbb{T}^{1} \times I .
\end{aligned}
$$

$\dagger$ For the sake of simplicity, we state the conditions in terms of derivatives, although the system may not be differentiable everywhere. Then these conditions should always be understood in the way that the mentioned inequalities hold for the liminf and limsup of the respective difference quotients. 

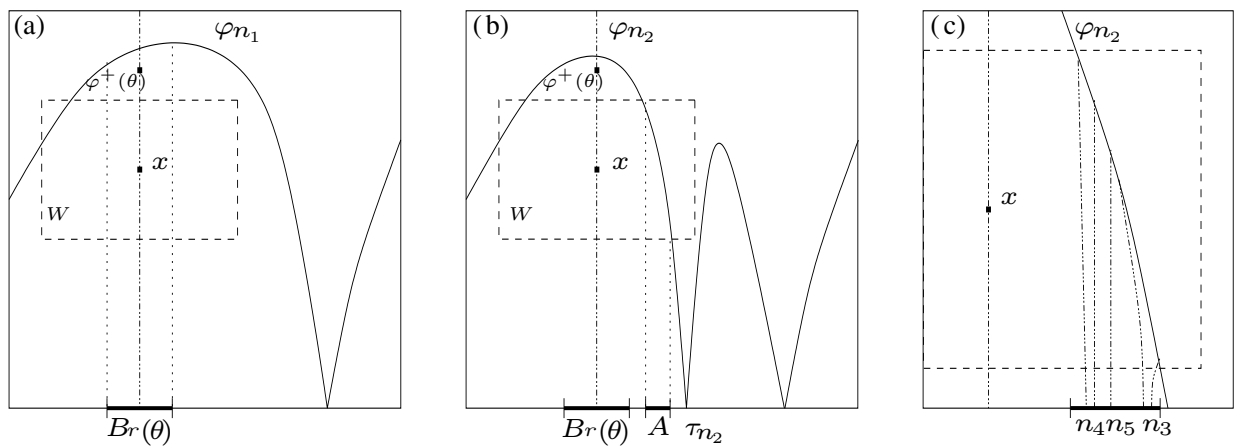

FIGURE 3. Taking the behaviour described in the text for granted, we could argue as follows. (a) Choose any $x \in\left(0, \varphi^{+}(\theta)\right)$ and any small box $W$ around $(\theta, x)$ which is below $\varphi^{+}(\theta)$. Fix $n_{1} \in \mathbb{N}$, such that the width of the peaks appearing at step $n_{1}$ or later is already small compared to $W$. As $\varphi_{n_{1}}(\theta) \geq \varphi^{+}(\theta)$ and due to the continuity of $\varphi_{n_{1}}$, this graph will be above $W$ on a whole neighbourhood $B_{r}(\theta)$ of $\theta$. (b) Now let $n_{2}$ be the first time greater than $n_{1}$ a peak comes so close to $B_{r}(\theta)$ that the value of the iterated upper boundary lines changes inside of $B_{r}(\theta)$. If there is one end of that peak which is contained in $B_{r}(\theta)$ (for now we ignore the other case, it can be treated similarly), then it will still be above $W$. On the other hand, $\varphi_{n_{2}}$ is pinched at $\tau_{n_{2}}$. Thus, $\varphi_{n_{2}}$ crosses $W$ on an interval $A$, and as the slope of $\varphi_{n_{2}}$ is at most $\beta \alpha^{n_{2}}$ for some $\alpha>1, \beta>0$ this interval still has a certain length. (c) A closer look at what happens in the interval $A$ after $n_{2}$. As it takes a long time until the next peaks hit this interval and the width of these peaks decays exponentially, they will not cover all of $A$. (Only the first three of these peaks at times $n_{3}, n_{4}$ and $n_{5}$ are depicted.) However, this means that $\varphi_{n_{2}}$ already coincides with $\varphi^{+}$at least for some $\theta^{\prime} \in A$ (in fact, on a Cantor set of positive measure). Therefore, $\Phi^{+} \cap W \neq \emptyset$, and as this works for any such box $W$ we can conclude $(\theta, x) \in \overline{\Phi^{+}}$.

Finally, assume there exists $m \in \mathbb{N}$ and $a, b>0$ with

$$
\begin{aligned}
m & >4+\frac{4}{\gamma}, \\
a & \geq(m+1)^{d}, \\
b & \leq c, \\
d\left(\tau_{n}, 0\right) & \geq b \quad \text { for all } n=1, \ldots, m-1,
\end{aligned}
$$

such that the following holds:

$$
T_{\theta}(x) \geq \min \{1, a x\} \cdot \min \left\{1, \frac{2}{b} d(\theta, 0)\right\} \quad \text { for all }(\theta, x) \in \mathbb{T}^{1} \times I .
$$

Remark 3.1. Here the reference system $R$ with fibre maps $R_{\theta}(x)=\min \{1, a x\}$. $\min \{1,(2 / b) d(\theta, 0)\}$ is only used implicitly as a lower bound for the original system. However, it should be mentioned that this simplified system proved to be very useful in the development of the presented ideas. Actually, all results were first derived in this simpler setting, where the heuristics described above can be directly converted into a rigorous proof.

Note also that (3.3) and (3.9) together imply a certain 'expansion-contraction property'. By (3.9) the system is expanding close to the 0-line and by (3.3) it is contracting further away. As we will see, the expansion is responsible for the fact that the peaks become steeper whereas the contraction will be needed to control what happens away from the peaks.

We start with two simple observations. 
LEMMA 3.2. We have the following.

(a) $\quad d\left(\tau_{n}, 0\right) \leq b \cdot a^{-i} \Rightarrow n \geq a^{i / d}\left(n \in \mathbb{Z}, i \in \mathbb{R}^{+}\right)$.

(b) $\left|\varphi_{n}^{\prime}\right| \leq \beta \cdot \alpha^{n}$.

Proof. (a) $d\left(\tau_{n}, 0\right) \leq b \cdot a^{-i} \stackrel{(3.1)}{\Rightarrow} c \cdot n^{-d} \leq b \cdot a^{-i} \stackrel{(3.7)}{\Rightarrow} n^{-d} \leq a^{-i} \Rightarrow n \geq a^{i / d}$.

(b) We prove $\left|\varphi_{n}^{\prime}\right| \leq \beta \cdot\left(\alpha^{n}-1\right)$ by induction. Note that

$$
\varphi_{n+1}^{\prime}(\theta)=D T_{\theta-\omega}\left(\varphi_{n}(\theta-\omega)\right) \cdot \varphi_{n}^{\prime}(\theta-\omega)+\frac{\partial}{\partial \theta} T_{\theta-\omega}\left(\varphi_{n}(\theta-\omega)\right)
$$

For $n=1$ the statement is obvious as $\varphi_{0}^{\prime} \equiv 0, \alpha>2$ and $\left|(\partial / \partial \theta) T_{\theta}(x)\right| \leq \beta((3.4))$. If it is satisfied for some $n \geq 1$, we get (from (3.2) and (3.4))

$$
\left|\varphi_{n+1}^{\prime}(\theta)\right| \leq \alpha \cdot\left|\varphi_{n}^{\prime}(\theta-\omega)\right|+\beta \leq \alpha \cdot \beta \cdot\left(\alpha^{n}-1\right)+\beta \leq \beta \cdot\left(\alpha^{n+1}-1\right),
$$

again using $\alpha>2$.

Let $J_{j}^{n}$ be an interval of length $b \cdot a^{-n / m}$ centred around $\tau_{j}$, i.e.

$$
J_{j}^{n}:=\left(\tau_{j}-\frac{b}{2} \cdot a^{-n / m}, \tau_{j}+\frac{b}{2} \cdot a^{-n / m}\right) .
$$

The following lemma now contains all of the information about the iterated upper boundary lines we need. The condition on $\theta$ is probably a little bit surprising at first. One might expect that the difference between $\varphi_{n-1}$ and $\varphi_{n}$ must be very small outside of a small interval around $\tau_{n}$. However, this is not entirely true. As we shall see, it is only possible to ensure that $\left|\varphi_{n-1}(\theta)-\varphi_{n}(\theta)\right|$ is small whenever $\theta$ is sufficiently far away from 'most' of the $\tau_{j}$, i.e. from $\tau_{q}, \tau_{q+1}, \ldots, \tau_{n}$ at the same time, where $q$ is relatively small compared to $n$. (The reason why we want to be able to omit the first few $\tau_{k}$ is going to become obvious later, when $q$ is specified in the proof of Theorem 4.1.) The proof below, together with Figure 4, hopefully clarifies why $\left|\varphi_{n-1}(\theta)-\varphi_{n}(\theta)\right|$ can be quite large not only when $\theta$ is close to $\tau_{n}$, but also when it is close to $\tau_{n-1}, \tau_{n-2}, \ldots$.

LEMMA 3.3. We have the following.

(a) Let $\theta \notin \bigcup_{j=q}^{n} J_{j}^{n-1}$. If $q \leq(n-1) / m$, then

$$
\left|\varphi_{n}(\theta)-\varphi_{n-1}(\theta)\right| \leq L \cdot \alpha^{-(n-1) \lambda}
$$

where $\lambda:=\gamma(1-4 / m)-4 / m$ is positive by (3.5).

(b) If $\theta \notin \bigcup_{j=q}^{\tilde{n}} J_{j}^{\tilde{n}-1} \cup \bigcup_{j=\tilde{n}+1}^{\infty} J_{j}^{j-1}$ and $q \leq(\tilde{n}-1) / m$, then (3.12) equally holds for all $n \geq \tilde{n}$.

(c) Let $\theta \notin\left\{\tau_{j} \mid j \in \mathbb{N}\right\}$. Then there are infinitely many $n \in \mathbb{N}$ such that $\theta \notin \bigcup_{j=1}^{n} J_{j}^{n-2}$. Note that the intervals used in (c) are slightly larger than those needed for the application of (a) to $\theta$. In fact, the difference is exactly $b / 2 \cdot\left(a^{-(n-2) / m}-a^{-(n-1) / m}\right)$ to either side. Therefore, (a) can be applied to all $\theta^{\prime}$ from a small neighbourhood of $\theta$. 
Proof. (a) Let $\theta_{k}:=\theta-n \omega+k \omega, x_{k}:=\varphi_{k}\left(\theta_{k}\right)$ and $s:=\# S$ where $S=\{1 \leq k<n \mid$ $\left.x_{k}<1\right\}$. Now (3.3) implies

$$
\begin{aligned}
\varphi_{n-1}(\theta)-\varphi_{n}(\theta)= & \left(\varphi_{0}\left(\theta_{1}\right)-\varphi_{1}\left(\theta_{1}\right)\right) \cdot \prod_{k=1}^{n-1} \frac{\varphi_{k}\left(\theta_{k+1}\right)-\varphi_{k+1}\left(\theta_{k+1}\right)}{\varphi_{k-1}\left(\theta_{k}\right)-\varphi_{k}\left(\theta_{k}\right)} \\
& \leq L \cdot \prod_{k=1}^{n-1} \underbrace{\frac{T_{\theta_{k}}\left(\varphi_{k-1}\left(\theta_{k}\right)\right)-T_{\theta_{k}}\left(\varphi_{k}\left(\theta_{k}\right)\right)}{\varphi_{k-1}\left(\theta_{k}\right)-\varphi_{k}\left(\theta_{k}\right)}}_{\leq \alpha^{-\gamma} \text { whenever } \varphi_{k}\left(\theta_{k}\right)=x_{k} \geq 1} \leq L \cdot \alpha^{s-\gamma(n-1-s)} .
\end{aligned}
$$

Thus, we are finished if we can show that $s \leq 4(n-1) / m$, because then $s-\gamma(n-1-s) \leq$ $(n-1)(4 / m-\gamma(1-4 / m))=-(n-1) \lambda$.

In order to obtain an estimate on $s$, we partition the set $S$ into blocks of the form $\{l+1, \ldots, p\}(0 \leq l<p<n)$ with the following properties:

(i) $x_{l} \geq 1 / a$;

(ii) $x_{k}<1 / a$ for all $k=l+1, \ldots, p-1$;

(iii) $x_{p}<1$;

(vi) either $x_{p} \geq 1 / a$ or $x_{p+1} \geq 1$ or $p+1=n$.

In other words, either two such blocks are separated by at least one time where $x_{k} \geq 1$, or we start a new block when the threshold $1 / a$ is reached. It is obvious that all such blocks give a partition of $S$ as required.

Now $\theta_{l} \in[-b / 2, b / 2]$ (otherwise $x_{l+1} \geq 1$ ), and the reference system (3.9) gives $x_{k+1} \geq a \cdot x_{k} \cdot \min \left\{1,(2 / b) d\left(\theta_{k}, 0\right)\right\}$ for all $k=l+1, \ldots, p-1$. As $x_{l} \geq 1 / a$ and $x_{p}<1$ we obtain

$$
\prod_{k=l}^{p-1} \min \left\{1, \frac{2}{b} d\left(\theta_{k}, 0\right)\right\}<a^{-(p-l-1)}
$$

This means that $\sum_{1 \leq i<\infty} i \cdot \#\left\{l \leq k<p \mid b / 2 \cdot a^{-i} \leq d\left(\theta_{k}, 0\right)<b / 2 \cdot a^{-i+1}\right\}$ is an upper bound on the length $p-l$ of the block (note that the inequality in (3.13) is strict), such that every visit $d\left(\theta_{k}, 0\right) \in\left[b / 2 \cdot a^{-i}, b / 2 \cdot a^{-i+10}\right)$ accounts for at most $i$ times $j>k$ where $x_{j}$ can be smaller than 1 . Summing this up for all blocks then gives an upper bound on $s$, namely

$$
s \leq \sum_{1 \leq i<\infty} i \cdot \#\left\{0 \leq k<n-1 \mid \frac{b}{2} \cdot a^{-i} \leq d\left(\theta_{k}, 0\right)<\frac{b}{2} \cdot a^{-i+1}\right\} .
$$

Now $\theta \notin \bigcup_{j=q}^{n} J_{j}^{n-1}$ implies that

$$
d\left(\theta_{k}, 0\right) \geq \frac{b}{2} \cdot a^{-(n-1) / m} \quad \text { for all } k=0, \ldots, n-q,
$$

anything else leads to the contradiction $\theta \in J_{n-k}^{n-1}$. In the worst case, $\tau_{n-q+1}$ is very close to 0 , 
such that $x_{n-q+1}, \ldots, x_{n-1}$ are all below 1 , but this still allows the estimate

$$
\begin{aligned}
s & \leq q-1+\sum_{1 \leq i \leq(n-1) / m+1} i \cdot \#\left\{0 \leq k \leq n-q \mid \frac{b}{2} \cdot a^{-i} \leq d\left(\theta_{k}, 0\right)<\frac{b}{2} \cdot a^{-i+1}\right\} \\
& \leq \frac{n-1}{m}-1+\sum_{1 \leq i \leq(n-1) / m+1} \#\left\{0 \leq k \leq n-q \mid d\left(\theta_{k}, 0\right)<\frac{b}{2} \cdot a^{-i+1}\right\} \\
& \stackrel{(*)}{\leq} \frac{2(n-1)}{m}+\sum_{2 \leq i \leq(n-1) / m+1} 1+\frac{n-1}{a^{(i-1) / d}} \leq \frac{3(n-1)}{m}+(n-1) \cdot \sum_{i=1}^{\infty} a^{-i / d} \\
& \stackrel{\text { (3.6) }}{\leq} \frac{4(n-1)}{m} .
\end{aligned}
$$

(*) Note that if $i \geq 2$, then $\theta_{k}$ can always visit the interval $\left(-b / 2 \cdot a^{-i+1}, b / 2 \cdot a^{-i+1}\right)$ once, but a second visit at time $l$ implies that $d\left(\theta_{k}, \theta_{l}\right)=d\left(\theta_{l-k}, 0\right)<b \cdot a^{-i+1}$ and thus $l-k \geq a^{(i-1) / d}$ by Lemma 3.2(a). Similarly, two visits in [-b/2,b/2] are at least $m$ times apart by (3.8).

(b) This is a direct consequence of (a).

(c) Let $n_{0} \in \mathbb{N}$ such that

$$
a^{(n-3) / m \cdot d} \geq n+1 \quad \text { for all } n \geq n_{0}
$$

and suppose that for some $k \geq n_{0}$ we have $\theta \in \bigcup_{j=1}^{k} J_{j}^{k-2}$. Let $\tau_{l}$ be the closest to $\theta$ of all points $\tau_{1}, \ldots, \tau_{k}$. Then there exists a unique $n>k$ such that $d\left(\theta, \tau_{l}\right) \in$ $\left[b / 2 \cdot a^{-(n-2) / m}, b / 2 \cdot a^{-(n-3) / m}\right)$. Now assume that for some $j>l$ we have $d\left(\tau_{j}, \theta\right)<$ $b / 2 \cdot a^{-(n-2) / m}$. This implies that $d\left(\tau_{j}, \tau_{l}\right)=d\left(\tau_{j-l}, 0\right)<b \cdot a^{-(n-3) / m}$ and therefore $j \geq j-l \geq a^{(n-3) / m \cdot d} \geq n+1$ by Lemma 3.2(a) and (3.14). Thus, $n$ satisfies $\theta \notin \bigcup_{j=1}^{n} J_{j}^{n-2}$ as required.

\section{The topological closure of the upper bounding graph}

Let us first reformulate the result of the last section. Fix some $\theta \in \mathbb{T}^{1}$ not contained in the forward orbit of the pinching point. When $\theta$ is inside of the $k$ th peak, then the value of $\varphi_{n}(\theta)$ may change significantly at time $n=k$ and for a certain time afterwards, but then the movement of the iterated upper boundary lines 'settles down' in a small neighbourhood of $\theta$, until the next peak visits that neighbourhood. Lemma 3.3(c) guarantees that there will always be such times where the $\varphi_{n}$ have 'settled down' close to $\theta$.

This can now be used to show that indeed $\overline{\Phi^{+}}=\left[0, \varphi^{+}\right]$, using a slight modification of the strategy sketched in Figure 3 . The peaks correspond to the intervals $J_{n}^{n-1} \cdot n_{2}$ will be chosen as before, i.e. the first time after $n_{1}$ at which the interval $J_{n_{2}}^{n_{2}-1}$ hits the neighbourhood $B_{r}(\theta)$. The main difference to Figure 3 is that we cannot control $\varphi_{n-1}-\varphi_{n}$ on $J_{n_{2}}^{n_{2}-1}$ until $n$ is larger than $n_{3}:=m\left(n_{2}+1\right)+1$ (see Lemma 3.3(a), where we will have to choose $q=n_{2}+1$ ). Thus, instead of looking at the graph $\varphi_{n_{2}}$ and showing that it coincides with $\varphi^{+}$for some $\theta^{\prime} \in B_{r}(\theta)$ (compare Figures 3(b) and (c)), we have to look at $\varphi_{n_{3}}$ and show that this graph is already very close to $\varphi^{+}$for some $\theta^{\prime} \in B_{r}(\theta)$.

It may be worth mentioning that the conditions (3.2)-(3.9) we put on our system only had to be used to obtain the statement of Lemma 3.3. From now on, this lemma contains everything we need to know about our system (apart from the pinched structure and the diophantine rotation number, of course). 


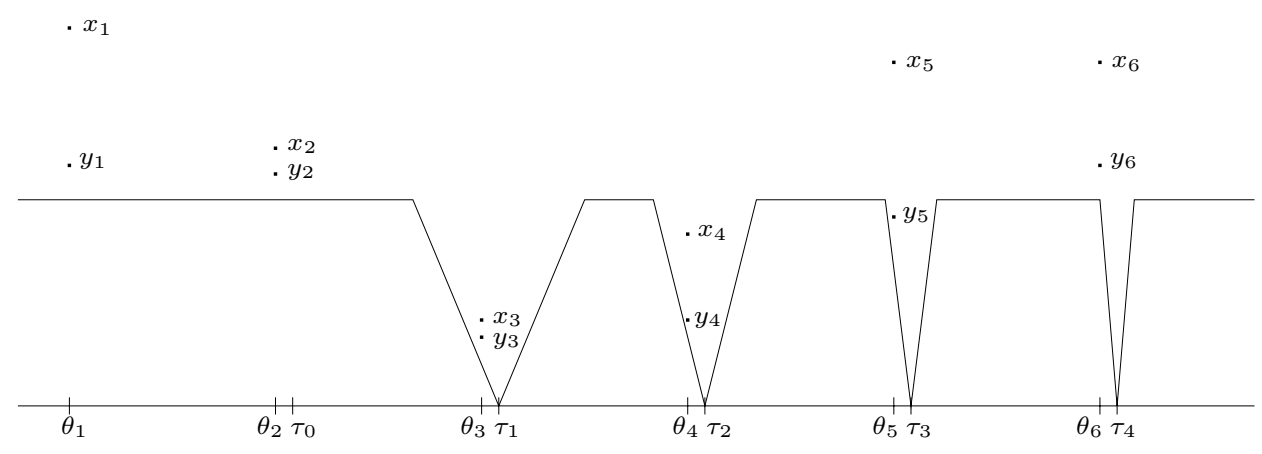

FIGURE 4. Why do we have to expect $\varphi_{n-1}(\theta)-\varphi_{n}(\theta)$ to be rather large not only when $\theta \in J_{n}^{n-1}$, but also when it is in $J_{j}^{n-1}$ for some $j<n$ ? We illustrate this with a simple example where $n=6$ and $\theta=\theta_{6}$ is close to $\tau_{4}$ (i.e. in $J_{4}^{5}$ ). Ignoring the fact that we are on the circle, the first four peaks of the reference system are drawn in a straight order. They give a lower bound for the points $y_{k}:=\varphi_{k-1}\left(\theta_{k}\right)$ and $x_{k}:=\varphi_{k}\left(\theta_{k}\right)$ $(k=1, \ldots, 6)$. In the first two steps these points are above 1 and their distance is contracted. However afterwards $\theta_{k}$ is always inside of the $(k-2)$ th peak, such that the points can be below 1 and their distance may be expanded again. Thus the assumption on $\theta$ in Lemma 3.3(a) is needed to exclude that any of the $\theta_{k}$ is too close to $\tau_{0}=0$, maybe apart from the last $q-1$ steps. The possibility that many different $\theta_{k}$ are close to $\tau_{0}$ is then ruled out by the diophantine condition on $\omega$.

THEOREM 4.1. Suppose T satisfies all of the assumptions (2.1), (2.2) and (3.1)-(3.9). Then

$$
\overline{\Phi^{+}}=\left[0, \varphi^{+}\right] .
$$

Proof. Suppose that $x \in\left(0, \varphi^{+}(\theta)\right)$ for some $\theta \in \mathbb{T}^{1}$. Choose $\epsilon>0$ such that $3 \epsilon \leq x \leq$ $\varphi^{+}(\theta)-3 \epsilon, \delta>0$ and consider boxes $W^{\prime}:=B_{\delta}(\theta) \times B_{2 \epsilon}(x), W:=B_{\delta}(\theta) \times B_{\epsilon}(x)$. It suffices to show that $W^{\prime} \cap \Phi^{+} \neq \emptyset$ for any such $\epsilon$ and $\delta$.

First of all, we will fix some $n_{0} \in \mathbb{N}$ which satisfies certain conditions. Roughly speaking, these imply that for all $n \geq n_{0}$ the intervals $J_{j}^{n-1}$ are small enough in comparison with $\delta$ and that the time $l$ we have to wait until $J_{j+l}^{n-1}$ hits $J_{j}^{n-1}$ again is very large. All of the conditions are obviously satisfied when $n_{0}$ is large enough. Apart from that the reader should be advised not to wonder about these requirements too long in the beginning, but to check each of them only at the time when it is actually used below and the motivation for choosing it becomes apparent. Let $n_{0} \in \mathbb{N}$ satisfy

$$
\begin{aligned}
n_{0} & \geq m+1 \\
\frac{b}{2} \cdot\left(a^{-(n-2) / m}-a^{-(n-1) / m}\right) & \leq \frac{\delta}{2} \quad \text { for all } n \geq n_{0} \\
\left|J_{j}^{n-1}\right|=b \cdot a^{-(n-1) / m} & \leq \frac{\delta}{2} \quad \text { for all } n \geq n_{0} \\
a^{(n-1) / m \cdot d} & >m(n+1)+1-n \quad \text { for all } n \geq n_{0} \\
\sum_{j \geq a^{(n-1) / m \cdot d}} b \cdot a^{-(j-1) / m} & \leq \frac{\epsilon}{\beta} \cdot \alpha^{-m(n+1)-1} \quad \text { for all } n \geq n_{0} \\
\sum_{j=n+1}^{\infty} L \cdot a^{-(j-1) \lambda} & \leq \epsilon \quad \text { for all } n \geq n_{0} .
\end{aligned}
$$


For (4.5) note that the left-hand side decays super-exponentially with $n$ whereas the righthand side only decays exponentially.

Now take some $n_{1} \geq n_{0}$ that satisfies

$$
\theta \notin \bigcup_{j=1}^{n_{1}} J_{j}^{n_{1}-2}
$$

Such an $n_{1}$ always exists by Lemma 3.3(c). It follows that

$$
\bigcup_{j=1}^{n_{1}} J_{j}^{n_{1}-1} \cap B_{r}(\theta)=\emptyset
$$

where $r:=b / 2 \cdot\left(a^{-\left(n_{1}-2\right) / m}-a^{-\left(n_{1}-1\right) / m}\right)>0$. As pointed out in Lemma 3.3(c), we can apply statement (a) of it to all $\theta^{\prime} \in B_{r}(\theta)$, and this is also possible for all $n \geq n_{1}$ as long as $B_{r}(\theta) \cap J_{j}^{j-1}=\varnothing$ for all $j=n_{1}+1, \ldots, n$. In addition we can assume, by reducing $r$ further if necessary, that $\varphi_{n_{1}}\left(\theta^{\prime}\right)>x+2 \epsilon$ for all $\theta^{\prime} \in B_{r}(\theta)$ (note that $\varphi_{n_{1}}$ is continuous and $\varphi_{n_{1}}(\theta) \geq \varphi^{+}(\theta) \geq x+3 \epsilon$ by the choice of $\left.\epsilon\right)$.

Let $n_{2}$ be the first integer greater than $n_{1}$ such that $J_{n_{2}}^{n_{2}-1}$ hits $B_{r}(\theta)$ and define $n_{3}:=m\left(n_{2}+1\right)+1$. (4.2) and (4.3) ensure that $J_{n_{2}}^{n_{2}-1} \subseteq B_{\delta}(\theta)$. Further, (4.4) ensures that

$$
\theta^{\prime} \notin \bigcup_{j=n_{2}+1}^{n_{3}} J_{j}^{j-1} \text { for all } \theta^{\prime} \in J_{n_{2}}^{n_{2}-1},
$$

because $J_{n_{2}}^{n_{2}-1} \cap J_{j}^{j-1} \neq \emptyset$ for some $j>n_{2}$ implies that $d\left(\theta_{j}, \theta_{n_{2}}\right) \leq b \cdot a^{\left(-n_{2}+1\right) / m}$, and as $n_{3}-n_{2}=m\left(n_{2}+1\right)+1-n_{2} \leq a^{\left(n_{2}-1\right) / m \cdot d}$ by (4.4) this cannot happen if $j-n_{2} \leq n_{3}-n_{2}$ by Lemma 3.2(a).

Now first assume that $\theta \in J_{n_{2}}^{n_{2}-1}$. As $\varphi_{n_{3}}$ is pinched at $\theta_{n_{2}}$ and at the same time $\varphi_{n_{3}}(\theta) \geq \varphi^{+}(\theta) \geq x+3 \epsilon$, this graph will cross the box $W$ from below to above when going from $\tau_{n_{2}}$ to $\theta$. Thus, if we define

$$
A:=\left\{\theta^{\prime} \in J_{n_{2}}^{n_{2}-1} \mid \varphi_{n_{3}}\left(\theta^{\prime}\right) \in B_{\epsilon}(x)\right\},
$$

we can use Lemma 3.2(b) to obtain that

$$
|A| \geq \frac{2 \epsilon}{\beta} \cdot \alpha^{-n_{3}}
$$

Let

$$
B:=A \backslash \bigcup_{j=n_{3}+1}^{\infty} J_{j}^{j-1}
$$

$B$ is still a set of positive measure: let $n_{4}$ be the first time where $J_{n}^{n-1}$ hits $J_{n_{2}}^{n_{2}-1}$ again. Then $n_{4} \geq a^{\left(n_{2}-1\right) / m \cdot d}$ (again Lemma 3.2(a)) and, therefore,

$$
\left|A \cap \bigcup_{j=n_{3}+1}^{\infty} J_{j}^{j-1}\right| \leq \sum_{j=n_{4}}^{\infty}\left|J_{j}^{j-1}\right| \leq \sum_{j \geq a^{\left(n_{2}-1\right) / m \cdot d}} b \cdot a^{-(j-1) / m} \stackrel{(4.5)}{\leq} \frac{\epsilon}{\beta} \cdot \alpha^{-n_{3}}
$$

where we used $n_{3}=m\left(n_{2}+1\right)+1$ in the last step. Thus, we have $|B| \geq \epsilon / \beta \cdot \alpha^{-n_{3}}$. 
Combining (4.9) and (4.11) we see that it is possible to apply Lemma 3.3(b) with $q=n_{2}+1$ and $\tilde{n}=n_{3}$ for every $\theta^{\prime} \in B$. Thus, the values of the graphs $\varphi_{n}\left(n \geq n_{3}\right)$ do not differ too much anymore on $B$, and by using $\varphi_{n} \searrow \varphi^{+}$we get

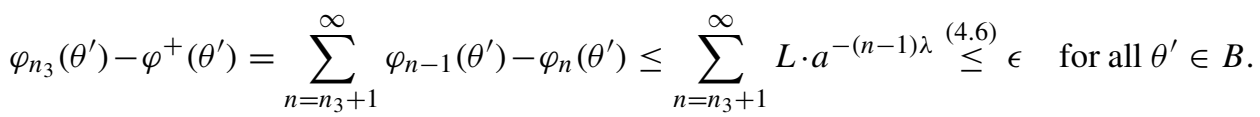

As $\varphi_{n_{3}}\left(\theta^{\prime}\right) \in B_{\epsilon}(x)$ for all $\theta^{\prime} \in B$ and $B \subseteq B_{\delta}(\theta)$ this proves that $\overline{\Phi^{+}} \cap W^{\prime} \neq \emptyset$.

When $\theta$ is not contained in $J_{n_{2}}^{n_{2}-1}$ we can equally conclude that $\varphi_{n_{3}}$ crosses $W$ inside of the interval $J_{n_{2}}^{n_{2}-1}$, such that the above argument applies in exactly the same way. To see this, note that when $\theta \notin J_{n_{2}}^{n_{2}-1}$, then at least one endpoint of $J_{n_{2}}^{n_{2}-1}$ is contained in $B_{r}(\theta)$. Denote it by $\bar{\theta}$. As $\bar{\theta} \notin J_{n_{2}}^{n_{2}-1}$ (recall that these intervals are open) we can combine (4.8), the definition of $n_{2}$ and (4.9) (which also applies to $\bar{\theta}$ as the endpoint of $J_{n_{2}}^{n_{2}-1}$ ) to obtain that $\bar{\theta} \notin \bigcup_{j=1}^{n} J_{j}^{n-1}$ for all $n=n_{1}, \ldots, n_{3}$, and as $n_{1} \geq m+1$ by (4.1) we can apply Lemma 3.3(a) with $q=1$ to obtain

$$
\varphi_{n_{3}}(\bar{\theta}) \geq \varphi_{n_{1}}(\bar{\theta})-\sum_{j=n_{1}+1}^{n_{3}} \varphi_{j-1}(\bar{\theta})-\varphi_{j}(\bar{\theta}) \geq x+2 \epsilon-\sum_{j=n_{1}+1}^{n_{3}} L \cdot a^{-(n-1) \lambda} \stackrel{(4.6)}{\geq} x+\epsilon
$$

Again $\varphi_{n_{3}}\left(\theta_{n_{2}}\right)=0$, such that the graph has to cross $W$ between $\theta_{n_{2}}$ and $\bar{\theta}$ and we can proceed as before. This completes the proof of the theorem.

Remark 4.2. In fact, the above proof even yields a slightly stronger statement than Theorem 4.1. To each invariant graph, an ergodic invariant measure $\mu_{\varphi}$ can be associated by

$$
\mu_{\varphi}(A):=m\left(\pi_{1}(\Phi \cap A)\right)
$$

i.e. by projecting the Lebesgue measure on $\mathbb{T}^{1}$ onto the graph. In the above proof we obtained $\mu_{\varphi^{+}}\left(W^{\prime}\right)>0$ for any open box $W^{\prime}$ around any point $(\theta, x) \in\left[0, \varphi^{+}\right]$. This allows us to conclude that not only $\overline{\Phi^{+}}$, but also the topological support of $\mu_{\varphi^{+}}$(see [KH97] for a definition) is equal to $\left[0, \varphi^{+}\right]$.

We now want to apply this to parameter families $T=T_{\alpha}$ with fibre maps given by (1.4). To that end, suppose that $g: \mathbb{T}^{1} \rightarrow \mathbb{T}^{1}$ and $f: \mathbb{R}^{+} \rightarrow \mathbb{R}^{+}$are continuous functions which satisfy the following assumptions:

$$
\begin{aligned}
& g \text { is differentiable and strictly positive on } \mathbb{T}^{1} \backslash\left\{\theta_{0}\right\}, \\
& \qquad g\left(\theta_{0}\right)=0, \\
& \lim _{\theta \nearrow \theta_{0}}-g^{\prime}(\theta)>0 \text { and } \lim _{\theta \searrow \theta_{0}} g^{\prime}(\theta)>0,
\end{aligned}
$$

$f$ is differentiable and monotonically increasing,

$$
\alpha^{1+\gamma} f^{\prime}(\alpha) \rightarrow 0(\alpha \rightarrow \infty) \text { for some } \gamma>0
$$

COROLlary 4.3. Suppose that $\omega$ satisfies a diophantine condition (3.1) and $T$ is given by

$$
(\theta, x) \mapsto(\theta+\omega, \alpha g(\theta) f(x))
$$


with maps $f$ and $g$ satisfying the above assumptions (4.12)-(4.17). Then there exists $\alpha_{0}>0$ such that

$$
\overline{\Phi^{+}}=\left[0, \varphi^{+}\right]
$$

whenever $\alpha \geq \alpha_{0}$.

Proof. Choose $m \geq 4+4 / \gamma$. Without loss of generality, we can assume that $\theta_{0}=0$ in (4.13). First note that the system

$$
(\theta, x) \mapsto\left(\theta+\omega, \alpha_{1} \alpha_{2} g(\theta) f(x)\right)
$$

is topologically conjugated to the system

$$
(\theta, x) \mapsto\left(\theta+\omega, \alpha_{1} g(\theta) f\left(\alpha_{2} x\right)\right)
$$

by $h:(\theta, x) \mapsto\left(\theta, \alpha_{2} x\right)$. Equations (4.12) and (4.14) and $f^{\prime}(0)>0$ imply that this system is bounded from below by a suitable reference system (3.9) if $\alpha_{1}$ and $\alpha_{2}$ are large enough. Once $\alpha_{1}$ is fixed, (4.17) implies that (3.3) is satisfied when $\alpha_{2}$ is sufficiently large. It is also easy to see that the system is pinched, has an invariant 0 -line, and that $(\partial / \partial \theta) T_{\theta}(x)$ is bounded for any $\alpha_{1}$ and $\alpha_{2}$. Thus, all of the assumptions of Theorem 4.1 are satisfied, and the conjugacy $h$ of course preserves the property we are interested in.

Example 4.4. Consider $g(\theta)=|\sin (\pi \theta)|, f(x)=\tanh (x)$ and let $\omega$ be the golden mean. The smallest possible value for $m$ in (3.5) is $m=5$ (if we can take $\gamma>4$, which we verify below). The closest return up to time $m-1=4$ is $\omega^{3} \approx 0.236 \ldots$, which we take for $b$. Now we just try whether $a=8$ works. To that end note that $3|\sin (\pi \theta)|>$ $\min \left\{1,\left(2 / \omega^{3}\right) d(0, \theta)\right\}$ and $\frac{4}{3} \tanh (1)>1$. Thus, $T_{\theta}(x)=4|\cos (\pi \theta)| \tanh (8 x)$ satisfies (3.9) with $a=8$ and $b=\omega^{3}$. As $\tanh ^{\prime}(x)=\cosh ^{-2}(x)<2 e^{-2 x}$, it is also easily checked that (3.3) holds for some $\gamma>4\left(2 e^{-16}<32^{-4}\right)$. Thus, we can choose $\alpha_{0}=32$ for this particular parameter family.

Compared to this, the lower bound for the existence of an SNA obtained from (2.4) is $\log \alpha>-\int_{\mathbb{T}^{1}} \log |\cos (\pi \theta)| d \theta$ or $\alpha>\exp \left(-\int_{\mathbb{T}^{1}} \log |\cos (\pi \theta)| d \theta\right)=2$, still leaving a certain gap between the two conditions. Of course, by suitable modifications of the assumptions and in the proofs above, this gap could be closed a little bit further, but doing so completely this way seems to be out of reach. However, as the approach used here requires a rather strong control about the behaviour of the systems, it does not seem very surprising that 'something gets lost along the way'. Thus, the existence of this gap does not necessarily have to mean that counterexamples should be expected for lower parameters $\alpha$. After all, there does not seem to be a qualitative change in the behaviour of the iterated upper boundary lines for smaller $\alpha$ (consider Figure 2 for example), as long as the upper bounding graph is not equal to 0 anyway.

Remark 4.5. When looking at pictures from numerical simulations as in Figure 1, the result we obtained may seem a little bit surprising at first. There, the larger we choose the parameter $\alpha$, the 'thinner' the SNA seems to be around the 0-line. However, our approach offers a perfect explanation for this. If $\alpha$ is large, the peaks will become steeper and narrower very fast. Thus, they will be too small to be detected numerically quite soon, and the SNA already seems to coincide with the iterated upper boundary line after a few steps. 
On the other hand, as we have already seen, it is exactly this fast decay of the width of the peaks which enabled us to prove our result.

The following remark contains a rather informal discussion of two possible slight generalizations of Theorem 4.1 and its corollary. We refrained from including them in the statement of the theorem, because although the basic idea does not change at all, this would have made the proof far less readable. The most prominent examples are already covered anyway.

Remark 4.6. (a) First, the diophantine condition on $\omega$ can be weakened to some extent. It was used to ensure that the return time to an interval of length $b \cdot a^{-i}$ grows exponentially in $i$ (see Lemma 3.2(a)), but actually all we need is that this quantity grows faster than linearly. This is the case as long as the rotation number $\omega$ satisfies $d\left(0, \tau_{n}\right) \geq s \cdot e^{-\sqrt[d]{n}}$ for some $s>0, d>1$ (then the return times will asymptotically grow at least like $i^{d}$ ). The requirements on the other parameters will certainly have to be stronger in this case, but apart from that the proof above needs only the slightest modifications.

(b) Second, instead of working with the reference system (3.9), it is also possible to use a system of the kind $R_{\theta}(x)=\min \{1, a x\} \cdot \min \left\{1,\left(b^{-2} d(\theta, 0)\right)^{p}\right\}$, i.e. to replace the 'sharp peak' at $\theta=0$ with a critical point of finite order $p$. Then, instead of counting the visits in the intervals $\left(-(b / 2) a^{-i},(b / 2) a^{-i}\right)$ one would have to counts the visits in $\left(-\left((b / 2) a^{-i}\right)^{1 / p},\left((b / 2) a^{-i}\right)^{1 / p}\right)$ (compare the proof of Lemma 3.3(a)). The diophantine condition (or that mentioned in (a)) still guarantees that the resulting sums converge, only the conditions on the parameters must certainly be altered again. In particular, note that such a $p$ always exists when $T$ is as in Corollary 4.3 and instead of satisfying (4.14), $g$ is real-analytic, as it then cannot have an infinitely degenerate critical point.

\section{Upper bounding graphs which contain isolated points}

For the construction of counterexamples, we need some facts about the combinatorics of irrational rotations, as they can for example be found in [dMvS93]. Let $\left(a_{n}\right)_{n \in \mathbb{N}}$ be the coefficients of the continued fraction expansion of $\omega,\left(q_{n}\right)_{n \in \mathbb{N}}$ the closest return times and $\left(\sigma_{n}\right)_{n \in \mathbb{N}}$ the closest returns. Then the following equations hold

$$
\begin{gathered}
\sigma_{n}=q_{n} \omega \bmod 1=\tau_{q_{n}}, \\
q_{0}=1, \quad q_{1}=a_{1}, \quad q_{n+1}=a_{n+1} q_{n}+q_{n-1}, \\
\sigma_{0}=\omega, \quad \sigma_{1}=1-a_{1} \omega, \quad \sigma_{n-1}=a_{n+1} \sigma_{n}+\sigma_{n+1}, \\
\frac{1}{q_{n+2}} \leq \sigma_{n} \leq \frac{1}{q_{n+1}} .
\end{gathered}
$$

For the remainder of this section we will assume that

$$
\sum_{i=0}^{\infty} \frac{q_{i}}{q_{i+1}}<\infty
$$

As $1 / 2 a_{i+1} \leq q_{i} / q_{i+1} \leq 1 / a_{i+1}$ (see (5.2)) this is equivalent to

$$
\sum_{i=1}^{\infty} \frac{1}{a_{i}}<\infty
$$




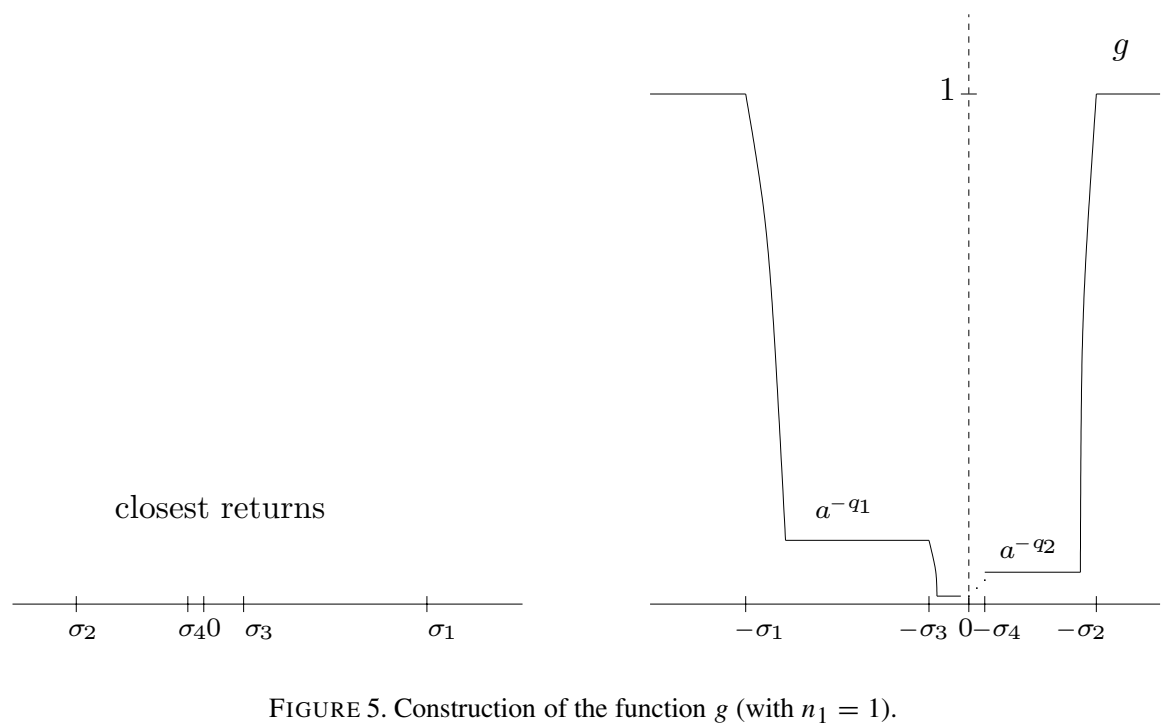

The examples we construct now will be of a similar form as in (1.4). The crucial point is the construction of a suitable function $g$.

Construction of $g$. Choose $n_{1} \in \mathbb{N}$ such that $\sum_{i=n_{1}}^{\infty} q_{i} / q_{i+1}<\frac{1}{2}$ and, for the sake of simplicity, $\sigma_{n_{1}}$ is to the right of 0 (in a local sense). Then all points $\sigma_{n_{1}+2 i}(i \in \mathbb{N})$ will be to the right of 0 , whereas all points $\sigma_{n_{1}+2 i+1}(i \in \mathbb{N})$ will be to the left. Let

$$
I_{n}^{\prime}:= \begin{cases}{\left[-\sigma_{n},-\sigma_{n+2}\right]} & \text { if } n-n_{1} \text { is even, } \\ {\left[-\sigma_{n+2},-\sigma_{n}\right]} & \text { if } n-n_{1} \text { is odd }\end{cases}
$$

and $I:=\bigcup_{n=n_{1}}^{\infty} I_{n}^{\prime} \cup\{0\}=\left[-\sigma_{n_{1}},-\sigma_{n_{1}+1}\right]$. Further, define intervals $I_{n} \subseteq I_{n}^{\prime}$ by

$$
I_{n}:= \begin{cases}{\left[-\sigma_{n}+\sigma_{n+2},-\sigma_{n+2}\right]} & \text { if } n-n_{1} \text { is even, } \\ {\left[-\sigma_{n+2},-\sigma_{n}+\sigma_{n+2}\right]} & \text { if } n-n_{1} \text { is odd. }\end{cases}
$$

Now choose any $a>2$ and a function $g$ with the property that $g_{\mid I_{n}} \equiv a^{-q_{n}}$ and $g_{\mid I_{n}^{\prime} \backslash I_{n}}$ joins the two levels $a^{-q_{n}}$ on $I_{n}$ and $a^{-q_{n-2}}$ on $I_{n-2}$ in a continuous and monotone way (such that $g\left(I_{n}^{\prime}\right)=\left[a^{-q_{n}}, a^{-q_{n-2}}\right]$ ). Similarly, we require $g \equiv 1$ outside of $I$ and join the two levels 1 on $I^{c}$ and $a^{-q_{n_{1}}}$ on $I_{n_{1}}$ by a suitable choice of $g$ on $I_{1}^{\prime} \backslash I_{1}$ (see Figure 5). Note that $|\log g|$ is integrable:

$$
\int_{\mathbb{T}^{1}}|\log g(\theta)| d \theta \leq \log a \cdot \sum_{i=n_{1}}^{\infty} q_{i} \cdot\left|\sigma_{i}\right| \stackrel{(5.4)}{\leq} \log a \cdot \sum_{i=n_{1}}^{\infty} \frac{q_{i}}{q_{i+1}} \leq \frac{1}{2} \log a .
$$

Further let $f(x):=\min \{1, a x\}$ and consider the system $T$ given by

$$
T:(\theta, x) \mapsto(\theta+\omega, g(\theta) f(x)) .
$$


Claim 1. We claim that

$$
\varphi^{+}(0)=1 .
$$

Proof. First of all $T_{\theta}(x) \leq 1$ for all $(\theta, x)$ by the choice of $f$ and $g$, such that $\varphi^{+}(0) \leq 1$. Therefore, it suffices to show $\varphi^{+}(0) \geq 1$.

Note that $\varphi_{n}(\theta) \neq \varphi_{n-1}(\theta)$ requires $\varphi_{n-1}(\theta-\omega) \neq \varphi_{n-2}(\theta-\omega)$, and in our particular example also $\varphi_{n-1}(\theta-\omega)<1 / a$. Let $n$ be the first time that $\varphi_{n}(0)<1$ (so that $n \geq 2$ as $\left.\tau_{-1} \notin I\right)$. Then necessarily $\varphi_{n-j}\left(\tau_{-j}\right)<1 / a$ for all $j=1, \ldots, n-1$. Thus, for the first $n-1$ steps the backwards orbit of $\left(0, \varphi_{n}(0)\right)$ is always in the expanding region and therefore

$$
\varphi_{n}(0)=T_{\tau_{-n}}^{n}(1)=a^{n-1} \cdot \prod_{j=1}^{n} g\left(\tau_{-j}\right) .
$$

As $\varphi_{n}(0)<1$, this implies that

$$
\prod_{j=1}^{n} g\left(\tau_{-j}\right)<a^{-(n-1)}
$$

However, we can estimate $-\log _{a} \prod_{j=1}^{n} g\left(\tau_{-j}\right)$ simply by counting how often $\left(\tau_{-j}\right)_{j=1, \ldots, n}$ visits the intervals $I_{i}^{\prime}$. As the time between two such visits must be at least $q_{i+1}$ $\left(\left|I_{i}^{\prime}\right|<\left|\sigma_{i}\right|\right)$, this gives

$$
-\log _{a} \prod_{j=1}^{n} g\left(\tau_{-j}\right) \leq \sum_{i=n_{1}}^{\infty} q_{i} \cdot \frac{n}{q_{i+1}} \leq \frac{n}{2} .
$$

As mentioned above, we can assume that $n \geq 2\left(\tau_{-1} \notin I\right)$ and thus arrive at a contradiction. This proves Claim 1.

Claim 2. We claim that

$$
\varphi^{+}(\theta) \leq \frac{1}{a} \quad \text { for all } \theta \in I \backslash\{0\} .
$$

Proof. This follows directly from the properties of $g$. When $n-n_{1}$ is even we have

$$
\varphi_{q_{n}} \leq a^{-q_{n}} \cdot a^{q_{n}-1}=\frac{1}{a} \quad \text { on }\left[-\sigma_{n}+\sigma_{n+2}, 0\right]+q_{n} \omega=\left[\sigma_{n+2}, \sigma_{n}\right]
$$

and $\bigcup_{i=0}^{\infty}\left[\sigma_{n_{1}+2 i+2}, \sigma_{n_{1}+2 i}\right]=\left(0, \sigma_{n_{1}}\right]$. The same argument applies to the left-hand side, which proves Claim 2.

The two claims together imply that $\left(0, \varphi^{+}(0)\right)=(0,1)$ is isolated in $\overline{\Phi^{+}}$, and by continuity of $T$ the same is true for all points in the backwards orbit of this point.

Remark 5.1. We have seen now that the topological closure of the upper bounding graph can have 'holes', namely on the fibres of the isolated points. However, as they are isolated these points will not be contained in the support of the associated measure $\mu_{\varphi^{+}}$, and it could certainly happen that this set is filled-in again (compare Remark 4.2). In this sense, the examples we have given must be considered rather 'weak' counterexamples. It would be interesting whether the topological support of the associated measure can have holes as well, or even to see examples of minimal sets which are not filled in, but to the knowledge of the author the existence of such examples is still a completely open question. 
Remark 5.2. We close with some final remarks concerning the regularity of the examples just constructed. First of all, it is possible to replace the rather degenerate function $f$ by a differentiable and strictly monotonically increasing function $\tilde{f}$. To that end, suppose that $\tilde{f}:[0,2] \rightarrow[0,2]$ satisfies $\tilde{f}_{\mid[0,1 / a]} \equiv f_{\mid[0,1 / a]}, \tilde{f}^{\prime}>0$ and $\tilde{f}(2) \leq 2$. Then the system

$$
\tilde{T}:(\theta, x) \mapsto(\theta+\omega, g(\theta) \tilde{f}(x))
$$

is bounded from below by (5.9), which ensures that $\tilde{\varphi}^{+}(0) \geq 1$. On the other hand, the same arguments as in the proof of Claim 2 show that $\tilde{\varphi}^{+}(\theta) \leq 2 / a<1$ for all $\theta \in\left[\sigma_{n_{1}+1}, \sigma_{n_{1}}\right]$.

Finally, although (5.6) certainly rules out the golden mean or any rotation number with bounded coefficients $a_{n}$, it still allows $\omega$ to be of diophantine type. Then it is possible to show, by combining the diophantine condition (3.1) with some elementary estimates obtained from (5.2)-(5.4), that $a^{-q_{n-2}} / \theta_{n+2}$ converges to zero as $n$ goes to infinity. Thus, the connecting pieces of $g$ on $I_{n}^{\prime} \backslash I_{n}$ can be chosen such that $g^{\prime}(\theta) \rightarrow 0$ as $\theta \rightarrow 0$, in which case $g$ is even differentiable. (However, more or less the same estimates yield that $g$ will then be infinitely flat at $\theta=0$, such that it cannot be bounded from below by any suitable reference system, compare Remark 4.6(b)).

Altogether, this means that the examples above can be chosen smooth and with a diophantine rotation on the base. Thus, additional assumptions on the geometry of the system as made in Theorem 4.1 cannot be omitted.

Acknowledgements. I thank Gerhard Keller for introducing me to the problem, and for many helpful remarks and discussions concerning this manuscript. The thoughtful remarks of an anonymous referee also helped to improve the paper. I also acknowledge the support of the Deutsche Forschungsgemeinschaft. This research was supported by grant Ke-514/6-1 of the German Science Foundation (DFG).

\section{REFERENCES}

[BO96] Z. I. Bezhaeva and V. I. Oseledets. An example of a strange non-chaotic attractor. Funct. Anal. Appl. 30(4) (1996), 223-229.

[dMvS93] W. de Melo and S. van Strien. One-dimensional Dynamics. Springer, Berlin, 1993.

[Gle02] P. Glendinning. Global attractors of pinched skew products. Dyn. Syst. 17 (2002), 287-294.

[GOPY84] C. Grebogi, E. Ott, S. Pelikan and J. A. Yorke. Strange attractors that are not chaotic. Physica D 13 (1984), 261-268.

[Her83] M. R. Herman. Une méthode pour minorer les exposants de Lyapunov et quelques exemples montrant le caractère local d'un théorème d'Arnold et de Moser sur le tore de dimension 2. Comment. Math. Helv. 58 (1983), 453-502.

[Jäg03] T. Jäger. Quasiperiodically forced interval maps with negative Schwarzian derivative. Nonlinearity 16(4) (2003), 1239-1255.

[Kel96] G. Keller. A note on strange nonchaotic attractors. Fund. Math. 151(2) (1996), 139-148.

[KH97] A. Katok and B. Hasselblatt. Introduction to the Modern Theory of Dynamical Systems. Cambridge University Press, Cambridge, 1997.

[PNR01] A. Prasad, S. S. Negi and R. Ramaswamy. Strange nonchaotic attractors. Internat. J. Bifur. Chaos 11(2) (2001), 291-309. 
[SS00] J. Stark and R. Sturman. Semi-uniform ergodic theorems and applications to forced systems. Nonlinearity 13(1) (2000), 113-143.

[Sta99] J. Stark. Regularity of invariant graphs for forced systems. Ergod. Th. \& Dynam. Sys. 19(1) (1999), 155-199.

[Sta03] J. Stark. Transitive sets for quasiperiodically forced monotone maps. Dyn. Sys. 18(4) (2003), 351-364. 